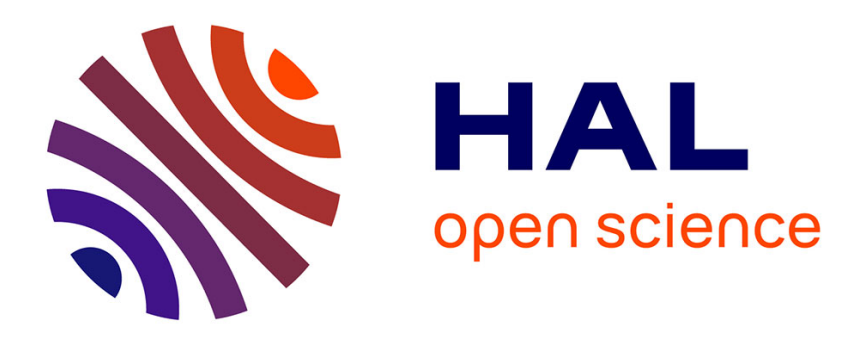

\title{
A Hybrid Systems Model for Power Control in Multicell Wireless Data Networks
}

\author{
Tansu Alpcan, Tamer Basar
}

\section{To cite this version:}

Tansu Alpcan, Tamer Basar. A Hybrid Systems Model for Power Control in Multicell Wireless Data Networks. WiOpt'03: Modeling and Optimization in Mobile, Ad Hoc and Wireless Networks, Mar 2003, Sophia Antipolis, France. 8 p. inria-00466298

\section{HAL Id: inria-00466298 https://hal.inria.fr/inria-00466298}

Submitted on 23 Mar 2010

HAL is a multi-disciplinary open access archive for the deposit and dissemination of scientific research documents, whether they are published or not. The documents may come from teaching and research institutions in France or abroad, or from public or private research centers.
L'archive ouverte pluridisciplinaire HAL, est destinée au dépôt et à la diffusion de documents scientifiques de niveau recherche, publiés ou non, émanant des établissements d'enseignement et de recherche français ou étrangers, des laboratoires publics ou privés. 


\title{
A Hybrid Systems Model for Power Control in Multicell Wireless Data Networks
}

\author{
Tansu Alpcan ${ }^{1}$ and Tamer Başar ${ }^{1}$ \\ (alpcan, tbasar)@control.csl.uiuc.edu
}

\begin{abstract}
We present a power control scheme based on noncooperative game theory, using a fairly broad class of convex cost functions. The multicell CDMA wireless data network is modeled as a switched hybrid system where handoffs of mobiles between different cells correspond to discrete switching events between different subsystems. Under a set of sufficient conditions, we prove the existence of a unique Nash equilibrium for each subsystem, and prove global exponential stability of an update algorithm. We also establish the global convergence of the dynamics of the multicell power control game to a convex superset of Nash equilibria for any switching (handoff) scheme satisfying a mild condition on average dwell-time. Robustness of these results to feedback delays as well as to quantization is investigated. In addition, we consider a quantization scheme to reduce the communication overhead between mobiles and the base stations. Finally, we illustrate the proposed power control scheme through simulations.
\end{abstract}

\section{INTRODUCTION}

The primary objective of power control in a wireless network is to regulate the transmission power level of each mobile in order to obtain and maintain a satisfactory quality of service for as many users as possible. In a code division multiple access (CDMA) system, where signals of other users can be modeled as interfering noise signals, the goal of power control is more precisely described as to achieve a certain signal to interference (SIR) ratio regardless of channel conditions while minimizing the interference, and hence improving the overall performance. Although there exists a large body of work for voice traffic where SIR requirements for satisfactory service are fixed and well established, power control for wireless data networks has only recently been a topic of interest [1], [2], [3], [4], [5], [6], [7]. Since the SIR requirements for a desired level of service vary from one individual user to another in wireless networks, the power control problem becomes also one of resource allocation. Recent studies [1], [4], [5] make use of concepts and tools from the field of economics, such as pricing and utility functions, to come up with power control schemes that address this question. In [4], a pricing scheme for the downlink of a wireless network is investigated where users are charged based on their channel quality. The study [5], on the other hand, shows that net utility maximization problem for elastic traffic can be decomposed into simpler problems of obtaining the optimal signal quality and selection of the optimal transmission rate.

\footnotetext{
${ }^{0}$ Research supported in part by the NSF grant CCR 00-85917.

${ }^{1}$ Coordinated Science Laboratory, University of Illinois, 1308 West Main Street, Urbana, IL 61801 USA.
}

In CDMA systems where each mobile interacts with others by affecting the SIR ratio through interference, game theory provides a natural framework for analyzing and developing power control mechanisms. For a mobile in such a network, obtaining individual information on the power level of each of the other users is practically impossible due to excessive communication and processing overhead required. Therefore in a distributed power control setting each user attempts to minimize its own cost (or maximize its utility) in response to the aggregate information on the actions of the other users. This makes use of noncooperative game theory [8] for uplink power control most appropriate, with the relevant solution concept being the noncooperative Nash equilibrium [9].

Several studies exist in the literature that use game theoretic schemes to address the power control problem in a single cell [3], [1]. In [9], we have made use of the conceptual framework of noncooperative game theory to obtain a distributed and market-based control mechanism. We have not only proven the existence of a unique Nash equilibrium, but also established the stability of two different update algorithms under specific conditions. Another recent study, [6], investigates pricing and power control in a multicell wireless network. Here, existence of a unique Nash equilibrium for a class of quasiconcave utility functions is established without pricing. The effect of linear pricing schemes on the solutions are also analyzed and it is shown that pricing improves Pareto efficiency of the operating (equilibrium) points.

In this paper, we extend the single cell power control scheme of [9] to multiple cells and to a broader class of cost functions. Specifically, we model the multicell wireless data network as a switched hybrid system where handoffs of mobiles between the individual cells (base stations) correspond to discrete switching events between different subsystems. Under a set of sufficient conditions, we show in Section II the existence and global stability of a unique Nash equilibrium for each subsystem. In Section III, we establish the global exponential convergence of the multicell power control game to a minimum convex set of Nash equilibria for any switching (handoff) scheme satisfying a mild condition on average dwell-time. Furthermore, we investigate robustness of these results to various communication constraints such as feedback delays and quantization. In addition, we analyze a quantization scheme to reduce the communication overhead between mobiles and the base stations. Finally, we illustrate the proposed power control scheme through MATLAB simulations in Section IV, which is followed by the concluding remarks of Section V. 


\section{The Model And Nash Equilibrium}

We consider a multicell CDMA wireless network model similar to the ones described in [6], [9]. The system consists of a set $\mathcal{L}:=\{1, \ldots, \bar{L}\}$ of cells, with $M_{l}$ users in cell $l$, $l \in \mathcal{L}$. The number of users in each cell is limited through an admission control scheme. We let $\mathcal{M}_{l}:=\left\{1, \ldots, M_{l}\right\}, l \in \mathcal{L}$. We associate a single base station (BS) with each cell in the system, and define $0<h_{i j}<1$ as the channel gain between the i-th mobile and the j-th BS. To simplify the analysis, we model secondary interference effects from neighboring cells as background noise, of variance $\sigma^{2}$. Furthermore, we assume that each mobile is connected to a single BS at any given time. Figure 1 shows a simple depiction of the wireless network model considered.

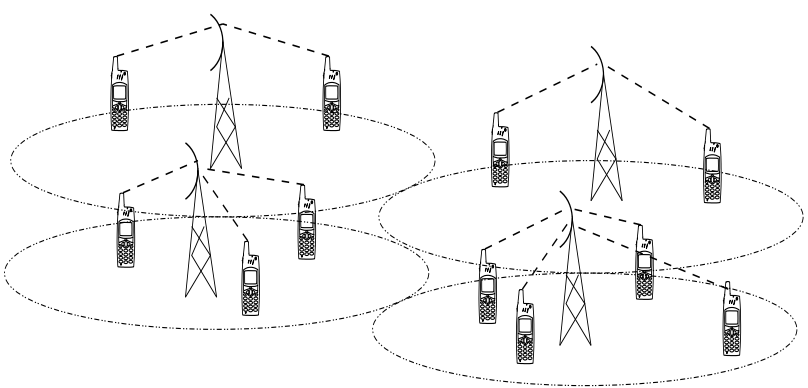

Fig. 1. A simple multicell wireless network.

The i-th mobile transmits with an uplink power level of $p_{i} \leq p_{i, \max }$, where $p_{i, \max }$ is an upper-bound imposed by physical limitations of the mobile. Thus, the SIR obtained by mobile $i$ at the base station $j$ is given by

$$
\gamma_{i j}:=\frac{L h_{i j} p_{i}}{\sum_{k \in \mathcal{M}_{j}, k \neq i} h_{k j} p_{k}+\sigma^{2}} .
$$

Here, $L:=W / R>1$ is the spreading gain of the CDMA system, where $W$ is the chip rate and $R$ is the data rate of the user. Define the power level of mobile $i$ received at the $\mathrm{j}$-th BS as $x_{i j}:=h_{i j} p_{i}$, and let $\mathbf{x}_{j}:=\left[x_{1 j}, \ldots, x_{M_{j} j}\right]$. Also let $\bar{x}_{-i j}:=\sum_{k \in \mathcal{M}_{j}, k \neq i} h_{k j} p_{k}$ be the sum of the received power levels of all mobiles, except the i-th one, at the j-th BS . In order to simplify the notation we will drop the index of the $\mathrm{BS}$ in the cases where we focus on a specific cell.

The i- $t h$ user's cost function is defined as the difference between the utility function of the user and its pricing function, $J_{i}=P_{i}-U_{i}$, similar to the one in [9]. The utility function, $U_{i}\left(\gamma_{i}\right)$, is a function of the SIR, $\gamma_{i}$, of the i-th user, and quantifies approximately the demand or willingness to pay of the user for bandwidth. The pricing function, $P_{i}\left(p_{i}\right)$, on the other hand, is imposed by the system to limit the interference created by the mobile, and hence improve the system performance [6]. At the same time, it can also be interpreted as a cost on the battery usage of the user. As a result, the cost function of the $\mathrm{i}-t h$ user connected to a specific $\mathrm{BS}$ is given by

$$
J_{i}\left(x_{i}, \mathbf{x}_{-i}, h_{i}\right)=P_{i}\left(x_{i}\right)-U_{i}\left(\gamma_{i}(\mathbf{x})\right),
$$

where we have used $x_{i}$, instead of $p_{i}$, as the argument of $P_{i}$, by a possible redefinition of the latter. We now make the following assumptions on the cost functions:

A1. $P_{i}\left(x_{i}\right)$ is twice continuously differentiable, nondecreasing and strictly convex in $x_{i}$, i.e.

$$
\partial P_{i}\left(x_{i}\right) / \partial x_{i} \geq 0, \partial^{2} P_{i}\left(x_{i}\right) / \partial x_{i}^{2}>0, \forall x_{i}
$$

A2. $U_{i}\left(\gamma_{i}(\mathbf{x})\right)$ is jointly continuous in all its arguments and twice continuously differentiable, nondecreasing and strictly concave in $x_{i}$, i.e. $\partial U_{i}(\mathbf{x}) / \partial x_{i} \geq 0, \partial^{2} U_{i}(\mathbf{x}) / \partial x_{i}^{2}<0, \forall x_{i}$.

A3. $U_{i}\left(\gamma_{i}\right)$ satisfies the inequality

$$
\left|\frac{d^{2} U_{i}}{d \gamma_{i}^{2}}\right| \gamma_{i}<\frac{d U_{i}}{d \gamma_{i}}<\left(L+\gamma_{i}\right)\left|\frac{d^{2} U_{i}}{d \gamma_{i}^{2}}\right|,
$$

where $|$.$| denotes the absolute value function.$

A4. The $i^{\text {th }}$ user's cost function has the following properties: At $x_{i}=0, J_{i}\left(\mathbf{x}: x_{i}=0\right)>J_{i}(\mathbf{x}), \forall \mathbf{x} x_{i} \neq 0$, and at $x_{i, \max }=x_{i, \max }, J_{i}\left(\mathbf{x}: x_{i}=x_{i, \max }\right)>J_{i}(\mathbf{x}), \forall \mathbf{x} x_{i}<$ $x_{i, \max }$, respectively.

Thanks to assumptions $A 1, A 2, A 3$, the cost function $J_{i}$ is strictly convex, and belongs to a fairly large subclass of convex functions. Hence, there exists a unique solution to the i-th user's minimization problem, which is that of minimization of $J_{i}$, given the system parameters and the power levels of other users. Since we have ignored secondary interference effects, a separate power control game is played in each of the $L$ cells in the system, and the Nash equilibrium (NE) in a cell is defined as a set of power levels, $\mathbf{p}^{*}$ (and corresponding set of costs $J^{*}$ ), with the property that no user in the cell can benefit by modifying its power level while the other players keep theirs fixed. Furthermore, assumption $A 4$ ensures that any equilibrium solution is an inner one, i.e., boundary solutions $x_{i}^{*}=0\left(x_{i}^{*}=x_{i, \max }\right) \quad \forall i$ cannot be equilibrium points. Mathematically speaking, $\mathrm{x}^{*}$ is in NE, when $x_{i}^{*}$ of any ith user is the solution to the following optimization problem given the equilibrium power levels of other mobiles in the same cell, $\mathbf{x}_{-i}^{*}$ :

$$
\min _{0 \leq x_{i} \leq x_{i, \max }} J_{i}\left(x_{i}, \mathbf{x}_{-i}^{*}, h_{i}\right) .
$$

Theorem II.1. Under A1-A4, the multicell power control game admits a unique inner Nash equilibrium solution.

Proof. The proof of this theorem is similar to the one of Theorem 3.1 in [10]. It is briefly outlined here for completeness. Let $X_{l}:=\left\{\mathbf{x} \in \mathbb{R}_{l}^{M}: 0 \leq \mathbf{x} \leq \mathbf{x}_{\max }\right\}$ be a set of feasible received power levels at the base station of a cell $l$ in the system, $l \in \mathcal{L}$. Clearly, $X_{l}$ is closed and bounded, and hence compact. Furthermore, it is also convex, and has a nonempty interior. By a standard theorem of game theory (Theorem 4.4 p.176 in [8]) the power control game in cell $l$ admits a Nash equilibrium. In addition, by $A 4$ this solution has to be inner. It follows from $A 3$ that

$$
\frac{\partial^{2} J_{i}}{\partial x_{i}^{2}}>\frac{\partial^{2} J_{i}}{\partial x_{i} \partial x_{j}}>0 .
$$

Finally, using an argument similar to the one in the proof of Theorem 3.1 in [10] one can show that the inner NE solution 
is unique in each cell. Thus, there exists a unique inner NE in the multicell power control game.

\section{HYBRID MODELING AND STABILITY}

We consider a dynamic model of the power control game where each mobile uses a gradient algorithm to solve its own optimization problem (3). The update scheme of the $i^{t h}$ mobile in a specific cell is given by

$$
\dot{p}_{i}=\frac{d p_{i}}{d t}=-\lambda_{i} \frac{\partial J_{i}}{\partial p_{i}}
$$

where $\lambda_{i}>0$ is a user-dependent stepsize. This can also be described in terms of the received power level, $x_{i}$, at the BS:

$$
\dot{x}_{i}=\frac{d U_{i}}{d \gamma_{i}} \frac{L \lambda_{i} h_{i}^{2}}{\sum_{j \neq i} x_{j}+\sigma^{2}}-\lambda_{i} h_{i} \frac{d P_{i}}{d p_{i}}:=\phi_{i}(\mathbf{x}) .
$$

Thus, we obtain a distributed power control algorithm which brings minimum overhead to the network for the only information the mobile needs in order to update its power level, other than its own most recent power level and the system parameters, is the level of total received power at the BS.

\section{A. Single Cell Network}

We first establish the stability of the update scheme (4) in a single cell $l$ under some sufficiency conditions. By taking the second derivative of $x_{i}$ with respect to time we obtain

$$
\ddot{x}_{i}=\left(-a_{i}-\frac{d^{2} P_{i}}{d p_{i}^{2}}\right) \dot{x}_{i}+\sum_{j \neq i} b_{i} \dot{x}_{j}:=\dot{\phi}_{i}(\mathbf{x})
$$

where $a_{i}$ and $b_{i}$ are defined as

$$
a_{i}:=\left|\frac{d^{2} U_{i}}{d \gamma_{i}^{2}}\right| \frac{L^{2} \lambda_{i} h_{i}^{2}}{\left(\sum_{j \neq i} x_{j}+\sigma^{2}\right)^{2}},
$$

and

$$
b_{i}:=\frac{a_{i}}{L} \gamma_{i}-\frac{d U_{i}}{d \gamma_{i}} \frac{L \lambda_{i} h_{i}^{2}}{\left(\sum_{j \neq i} x_{j}+\sigma^{2}\right)^{2}} .
$$

Notice that, $a_{i}$ is positive, and under $A 3, b_{i}$ is negative.

Let us introduce the candidate quadratic Lyapunov function

$$
V_{l}:=\sum_{i \in \mathcal{M}_{l}} \phi_{i}^{2}(\mathbf{x})
$$

As $\|\mathbf{x}\| \rightarrow \infty,\left(d U_{i} / d \gamma_{i}\right)\left(L \lambda_{i} h_{i}^{2}\right) /\left(\sum_{j \neq i} x_{j}+\sigma^{2}\right)$ is bounded by $A 2$ and $\left|\lambda_{i} h_{i} d P_{i} / d p_{i}\right| \rightarrow \infty$ by $A 1$. Hence, $V_{l}$ is radially unbounded, $V_{l} \rightarrow \infty \Leftrightarrow\|\mathbf{x}\| \rightarrow \infty$, where $\|$.$\| denotes the$ norm operator.

Taking the derivative of $V_{l}$ with respect to $t$ we have

$$
\dot{V}_{l} \leq \sum_{i \in \mathcal{M}_{l}}-2 a_{i} \phi_{i}^{2}+\sum_{i \in \mathcal{M}_{l}}\left|b_{i}\right| \sum_{j \in \mathcal{M}_{l}, j \neq i} 2\left|\phi_{i} \phi_{j}\right| .
$$

It follows from a simple algebraic manipulation that

$$
\sum_{i \in \mathcal{M}_{l}}\left|b_{i}\right| \sum_{j \in \mathcal{M}_{l}, j \neq i} 2\left|\phi_{i} \phi_{j}\right| \leq 2\left(M_{l}-1\right) \max _{i}\left|b_{i}\right| \sum_{i \in \mathcal{M}_{l}} \phi_{i}^{2} \text {. }
$$

Using this to bound $V_{l}$ further above yields

$$
\dot{V}_{l} \leq\left(-\min _{i} 2 a_{i}+2\left(M_{l}-1\right) \max _{i}\left|b_{i}\right|\right) \sum_{i \in \mathcal{M}_{l}} \phi_{i}^{2} .
$$

Next, we refine assumption $A 3$ as follows:

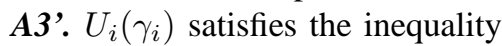

$$
\left|\frac{d^{2} U_{i}}{d \gamma_{i}^{2}}\right| \gamma_{i}<\frac{d U_{i}}{d \gamma_{i}}<\left(1+\gamma_{i}\right)\left|\frac{d^{2} U_{i}}{d \gamma_{i}^{2}}\right| .
$$

Remark III.1. A large class of logarithmic utility functions, $U_{i}=u_{i} \log \left(k \gamma_{i}+1\right)$, where $k>1$ and $u_{i}>0$ are scalar parameters, satisfy assumptions $A 2$ and $A 3$ '.

Under $A 3$ ', we have $0 \leq\left|b_{i}\right| \leq a_{i} / L$. Hence, a sufficient condition for $\dot{V}_{l}<0$, uniformly in the $x_{i}$ 's, is

$$
L>m_{l}\left(M_{l}-1\right)
$$

where $m_{l}$ is defined as

$$
m_{l}:=\max _{\mathbf{x} \in X_{l}} \frac{\max _{i \in \mathcal{M}_{l}} a_{i}}{\min _{i \in \mathcal{M}_{l}} a_{i}} .
$$

Thus, $V_{l}$ is indeed a Lyapunov function, and being also radially unbounded in $x_{i}$ 's, it readily follows that $\phi_{i}(\mathbf{x})=$ $\dot{x}_{i}(t) \rightarrow 0, \forall i$. This in turn implies that $x_{i}(t)$ 's converge to the unique Nash equilibrium. Hence, the unique NE point (Theorem II.1) of cell $l$ is globally asymptotically stable with respect to the update scheme (4) under the sufficient condition (6).

Remark III.2. In the symmetric case where $U_{i}=U_{j}$ and $x_{i}=$ $x_{j} \forall i, j \in \mathcal{M}_{l}$, the value of parameter $m_{l}$ is one. Therefore, the sufficient condition in (6) simplifies to

$$
L>M_{l}-1 \text {. }
$$

\section{B. Multiple Cell Network}

We next investigate the network-wide stability for a fixed number of mobiles by extending this result to multiple cells. Define the Lyapunov function

$$
V:=\sum_{l \in \mathcal{L}} V_{l}=\sum_{l \in \mathcal{L}} \sum_{i \in \mathcal{M}_{l}} \phi_{i}^{2} .
$$

Furthermore, assume that (6) holds for all $l \in \mathcal{L}$. This requirement can be satisfied by choosing $L$ sufficiently large and/or by implementing an admission control scheme at each cell to limit the maximum number of users, $M_{l}$. Therefore, we obtain $\dot{V}<0$. Thus, the unique NE of the given multicell power control game is globally asymptotically stable in the static case, where each mobile is connected to a specific base station (belongs to a cell) for all times. This result is stated in the following theorem:

Theorem III.3. Let $\mathbf{x}^{N E}:=\left[\mathbf{x}_{1}^{*}, \mathbf{x}_{2}^{*}, \ldots, \mathbf{x}_{L}^{*}\right]$ be the unique NE of a static multicell CDMA wireless network, where $\mathbf{x}_{i}^{*}:=$ $\left[x_{1 i}^{*}, x_{2 i}^{*}, \ldots, x_{M_{i} i}^{*}\right]$, and each mobile $i \in \mathcal{M}_{l}$ in cell $l \in \mathcal{L}$ stays connected to the respective $B S$ for all times. Then, the system is globally asymptotically stable if

$$
L>m_{l}\left(M_{l}-1\right), \forall l \in \mathcal{L} .
$$

In the static case there are no handoffs (switches) in the network. Consider now the dynamic case, where mobiles connect to base stations dynamically using criteria like SIR or channel gain as they move along the cells. Then, it is possible 
to consider each static case with a fixed distribution of users among cells as a separate subsystem, $q$, belonging to a family (set) of systems denoted by $Q$. This leads to a hybrid system where each handoff corresponds to switching from one system to another. In the study of this hybrid system we make use of the concept of (average) dwell-time, $\tau$, which quantifies the minimum (average) amount of time between two switches. In a wireless network handoffs are random in nature, and they may occur in short bursts. Hence, the concept of average dwell-time is much less restrictive than the dwell-time.

Based on our previous analysis for the static case, we define a quadratic Lyapunov function $V^{(q)}$ as in (7) for the subsystem $q \in Q$. Modifying condition (6) as

$$
L>m_{l}\left(M_{l}-1\right)+\epsilon, \forall l \in \mathcal{L},
$$

where $\epsilon>0$ is a positive constant yields

$$
\dot{V}^{(q)} \leq-\epsilon V^{(q)} .
$$

The unique NE, $\mathbf{x}_{q}^{N E}$, of the $q^{t h}$ subsystem is then globally exponentially stable by Theorem III.3.

To simplify the rest of the analysis, we will make the following assumption:

A5. In the multicell wireless network, no two handoffs can occur at the same time.

Since, under $A 5$, the times of occurence of multiple handoffs may still be arbitrarily close to each other, this technical assumption is not restrictive in practice. As a result of $A 5$, switching can happen only between "neighbor" subsystems, due to the handoff of a single mobile between two neighboring cells in the network. Hence, there exists a finite $\mu>1$ such that

$$
\frac{V^{(q)}}{V^{(r)}} \leq \mu
$$

where $q, r \in Q$ are any two "neighbor" subsystems. Let us also define the set of NE for all subsystems as

$$
\mathcal{N}:=\left\{\mathbf{x}_{q}^{N E}, \forall q \in Q\right\} .
$$

At a given time instance the system has only one NE which is an element of the set $\mathcal{N}$. However, this unique NE also switches from one equilibrium to the next in the set $\mathcal{N}$ with each handoff in the system.

We now make use of the results in Theorem 3.2 of [11] (Theorem 4 of [12]), a version of which is given here for completeness.

Theorem III.4. Consider a family of systems, $Q$, defined by $\dot{x}=f_{q}(x), \forall q \in Q$ with origin being the unique equilibrium. Let $V^{(q)}$ be a radially unbounded, quadratic Lyapunov function for any subsystem $q \in Q$ such that

$$
\dot{V}^{(q)}(x) \leq-\epsilon V^{(q)}(x) .
$$

Suppose also that there exists a finite $\mu>1$ such that

$$
\frac{V^{(q)}}{V^{(r)}} \leq \mu, \quad q, r \in Q
$$

Then, the switched system is globally exponentially stable for every switching signal with average dwell-time

$$
\tau>\frac{\log \mu}{\epsilon} \text {. }
$$

Notice that this theorem does not apply directly in our case as the equilibrium point of the multicell system shifts with each switching. We have, however, the following modified result:

Theorem III.5. Assume that the following condition holds for all the cells in the wireless network, for some $\epsilon>0$ :

$$
L>m_{l}\left(M_{l}-1\right)+\epsilon, \forall l \in \mathcal{L},
$$

where

$$
m_{l}:=\max _{\mathbf{x} \in X_{l}} \frac{\max _{i \in \mathcal{M}_{l}} a_{i}}{\min _{i \in \mathcal{M}_{l}} a_{i}} .
$$

Then, under the set of assumptions A1, A2, A3', A4, A5, the dynamics of the multicell power control game globally exponentially converge to the smallest convex set $\widehat{\mathcal{N}}$, which contains all possible $N E$ of the game $(\mathcal{N})$, for every handoff (switching) scheme with average dwell-time

$$
\tau>\frac{\log \mu}{\epsilon} .
$$

Furthermore, if we let $\overline{\mathcal{N}}$ be the smallest convex set that includes the union of the smallest level sets of $V^{(q)}, \forall q \in Q$ that contain $\widehat{\mathcal{N}}$, then the set $\overline{\mathcal{N}}$ is invariant under the same set of conditions.

Proof. It was previously shown that all the conditions in Theorem III.4 follow directly from (8). Hence, the system exponentially converges to the equilibrium, in this case, to the convex set $\widehat{\mathcal{N}} \supset \mathcal{N}$, if the condition on average dwelltime is satisfied. We omit the derivations as our proof follows basically the same lines as the ones in the proof of Theorem III.4. Further details can be found in [11]. Although the system is not asymptotically stable with respect to a single equilibrium point due to the shift of NE with switching, it converges globally, exponentially to the set $\widehat{\mathcal{N}}$.

We now show the invariance of $\overline{\mathcal{N}}$. Suppose that $\overline{\mathcal{N}}$ is not invariant. Then, there exists a trajectory that starts at a point $\mathbf{x} \in \overline{\mathcal{N}}$ and leaves this set. By definition, this would correspond to an increase in $V^{(q)}$ for some $q \in Q$, which leads to a contradiction. Thus, $\overline{\mathcal{N}}$ is invariant under the set of conditions of the theorem.

Remark III.6. By assumption A5, the equilibrium point of the system jumps from NE of a subsystem to the NE of a "neighbor" subsystem which is close in "distance" to it, where the distance is defined by a chosen norm. Hence, in a wireless network where distribution of mobiles change slowly over time the operating point of the system may stay in a subset of $\widehat{\mathcal{N}}$ over a time interval much longer than the update interval. In this case, the practical "size" of the set $\overline{\mathcal{N}}$ is much smaller than the one in the most general case. 
Proposition III.7. Consider a wireless network with of $M$ mobiles and $\bar{L}$ non-empty cells. If $M \rightarrow \infty$ then $\mu \rightarrow 1$, and hence, $\tau \rightarrow 0$.

Proof. Without loss of generality, assume that the $k^{\text {th }}$ mobile switches from a cell $m \in \mathcal{L}$ to cell $n \in \mathcal{L}$. Then, by definition of $\mu$,

$$
\mu=\frac{\Xi+\sum_{i \in \mathcal{M}_{m}} \phi_{i}^{2}+\sum_{i \in \mathcal{M}_{n}} \phi_{i}^{2}}{\Xi+\sum_{i \in \mathcal{M}_{m}, i \neq k} \bar{\phi}_{i}^{2}+\sum_{i \in \mathcal{M}_{n}} \bar{\phi}_{i}^{2}+\phi_{k}^{2}},
$$

where $\Xi=\sum_{l \in \mathcal{L}, l \neq m, n} \sum_{i \in \mathcal{M}_{l}} \phi_{i}^{2}$. As $M \rightarrow \infty$,

$$
\Xi \gg \sum_{i \in \mathcal{M}_{m}} \phi_{i}^{2}+\sum_{i \in \mathcal{M}_{n}} \phi_{i}^{2} \text { and } \bar{\phi}_{i}^{2} \rightarrow \phi_{i}^{2}, \forall i \in \mathcal{M}_{n}, \mathcal{M}_{m} .
$$

Hence, $\mu$ converges asymptotically to 1 . Therefore, by Theorem III.5 average dwell-time, $\tau$, diminishes to zero.

\section{Stability under Feedback Delays}

Even if we assume that mobiles have perfect information on their channel gain, cost, and system parameters, they still need the total received power level to be provided by the base station in order to implement the dynamic update algorithm (4). The BS determines the total received power level through measurements, and sends it to the mobile. Measurement, processing (both at $\mathrm{BS}$ and mobile), and signaling of this information takes time, which results in non-negligible delays in the network, which can go up to 0.5 seconds in GSM systems (see [7], p.28). Here we model such delays as a single fixed feedback delay. Since propagation delays are negligible for cellular wireless networks, all mobiles in a cell experience almost the same amount of feedback delay. In other words, delays are symmetric within a cell. Hence, the power update function of the $i^{t h}$ mobile in terms of $x_{i}$ becomes

$\dot{x}_{i}(t)=\frac{d U_{i}}{d \gamma_{i}} \frac{L \lambda_{i} h_{i}^{2}}{\sum_{j \neq i} x_{j}(t-r)+\sigma^{2}}-\lambda_{i} h_{i} \frac{d P_{i}}{d p_{i}}(t):=\varphi_{i}(\mathbf{x}(t))$,

where $r>0$ denotes the feedback delay in the network.

We now investigate stability of a single cell, $l$, by introducing the radially unbounded, quadratic Lyapunov function

$$
\begin{aligned}
& V_{l}(\mathbf{x}(t)):=\sum_{i \in \mathcal{M}_{l}} \phi_{i}^{2}(\mathbf{x}(t)) \\
& +\left(\max _{\mathbf{x} \in X_{l}} \max _{i}\left|b_{i}\right|\right)\left(M_{l}-1\right) \sum_{i \in \mathcal{M}_{l}} \int_{t-r}^{t} \phi_{i}^{2}(\mathbf{x}(s)) d s .
\end{aligned}
$$

Assuming $A 1, A 2, A 3$ ', and $A 4$ hold, we essentially repeat the Lyapunov analysis of Section III-A. Taking the derivative of $V_{l}(\mathbf{x}(t))$ with respect to $t$, we have

$$
\begin{aligned}
\dot{V}_{l}(\mathbf{x}(t)) \leq & \sum_{i \in \mathcal{M}_{l}}-2 a_{i} \phi_{i}^{2}(\mathbf{x}(t))+\left(\max _{\mathbf{x} \in X_{l}} \max _{i}\left|b_{i}\right|\right) \\
& {\left[\sum_{i \in \mathcal{M}_{l}} \sum_{j \in \mathcal{M}_{l}, j \neq i} 2\left|\phi_{i}(\mathbf{x}(t)) \phi_{j}(\mathbf{x}(t-r))\right|\right.} \\
& \left.+\left(M_{l}-1\right)\left(\phi_{i}(\mathbf{x}(t))^{2}-\phi_{i}(\mathbf{x}(t-r))^{2}\right)\right]
\end{aligned}
$$

It again follows from a simple algebraic manipulation that

$$
\begin{aligned}
& \sum_{i \in \mathcal{M}_{l}} \sum_{j \in \mathcal{M}_{l}, j \neq i} 2\left|\phi_{i}(\mathbf{x}(t)) \phi_{j}(\mathbf{x}(t-r))\right| \\
\leq & \left(M_{l}-1\right) \sum_{i \in \mathcal{M}_{l}} \phi_{i}^{2}(\mathbf{x}(t))+\phi_{i}^{2}(\mathbf{x}(t-r)) .
\end{aligned}
$$

Using this to bound $V_{l}$ further above yields

$\dot{V}_{l}(\mathbf{x}(t)) \leq\left(-\min _{i} 2 a_{i}+2\left(M_{l}-1\right)\right) \max _{\mathbf{x} \in X_{l}} \max _{i}\left|b_{i}\right| \sum_{i \in \mathcal{M}_{l}} \phi_{i}^{2}(t)$

Hence, a sufficient condition for $\dot{V}_{l}(\mathbf{x}(t))<0$, uniformly in the $x_{i}$ 's, is

$$
L>m_{l}\left(M_{l}-1\right)
$$

where $m_{l}$ is defined as

$$
m_{l}:=\frac{\max _{\mathbf{x} \in X_{l}} \max _{i \in \mathcal{M}_{l}} a_{i}}{\min _{\mathbf{x} \in X_{l}} \min _{i \in \mathcal{M}_{l}} a_{i}} .
$$

Thus, the unique NE point (Theorem II.1) of cell $l$ is globally asymptotically stable with respect to the update scheme (9) under the sufficient condition (10) for any feedback delay $r$. Note that the condition in (10) is more restrictive than the one in (6). Moreover, although the value of $r$ does not affect stability, large delays may result in slower convergence rates, and they may decrease the robustness of the system.

This result can be extended in a straightforward way to the static and dynamic multiple cell cases to obtain counterparts of Theorems III.3 and III.5 for the delayed information case.

\section{Communication Constraints}

Total received power level, $\sum_{i \in \mathcal{M}} x_{i}+\sigma^{2}$, at the BS of a cell constitutes the main information flow in the distributed power update scheme (4). BS has to send mobiles this quantity (state information) as frequently as possible in order for the update algorithm to converge. This, however, may bring a significant overhead to the system, if not implemented efficiently. We investigate here a simple practical scheme which lessens the communication overhead, and hence, increases the efficiency through quantization (see Figure 2).

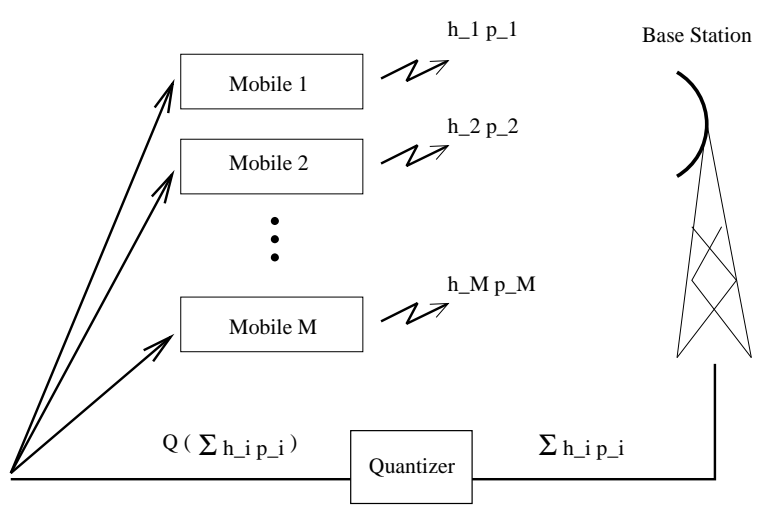

Fig. 2. A simple quantization scheme for reducing overhead in the system.

Although total received power level can be measured to a great accuracy at the $\mathrm{BS}$, it is not necessary to send this information to the mobile in its most accurate form as this 
would waste valuable bandwidth. Instead, this value can be quantized without destroying system stability. We consider, for its simplicity and ease of implementation, a uniform quantization scheme. Assume that there exists a fixed practical upper bound on $\sum_{i \in \mathcal{M}_{l}} x_{i}+\sigma^{2}$, defined as $F$ for any cell $l$. Then, a $K$ level quantization of aggregate received power level is

$$
0 \leq \theta_{K}\left(\sum_{i \in \mathcal{M}_{l}} x_{i}+\sigma^{2}\right) \leq F, \forall l \in L
$$

where $\theta_{K}$ is a $K$ level uniform quantizer. As long as $\sum_{i \in \mathcal{M}_{l}} x_{i}+\sigma^{2} \in[0, F]$ holds for all cells, the maximum quantization error $\xi$ is defined as $\xi:=F / 2 K$.

A derivation similar to the one in Section III-A results in the following modified version of the sufficient condition in (10) for stability of the $l^{\text {th }}$ cell under the existence of the quantization error $\xi$,

$$
L>\bar{m}_{l}\left(M_{l}-1\right),
$$

where $\bar{m}_{l}$ is defined as

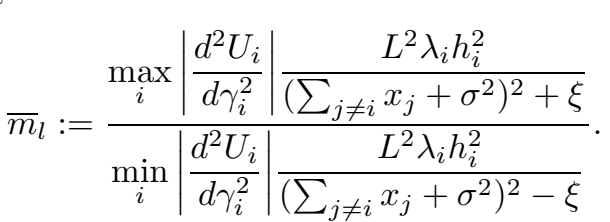

Hence, given a sufficiently large $L$, there exists an upperbound on $\xi$ which preserves stability. Using this value of $\xi$ we obtain the minimum number of bits to represent the feedback information,

$$
\text { bit }_{\text {min }}=\left\lceil\log _{2} F /(2 \xi)\right\rceil,
$$

where $\lceil$.$\rceil denotes ceiling function (rounding up to the next$ integer). Assume that the mobile update frequency is $f_{\text {update }}$ Hertz. Then, the system overhead in the downlink for a mobile is given by

$$
\text { bitrate }=f_{\text {update }}\left\lceil\log _{2} F /(2 \xi)\right\rceil \text { bits-per-second (bps). }
$$

The rate of change in the total received power level in a cell can be bounded above by $F_{v a r}$,

$$
\frac{\partial\left(\sum_{i \in \mathcal{M}} x_{i}+\sigma^{2}\right)}{\partial t} \leq F_{v a r} .
$$

Therefore, sending the mobiles the incremental changes in the total received power level instead of sending the whole information each time results in bandwidth savings. In this case, in order to maintain the given maximum quantization error, $\xi$, the minimum number of bits to use is

$$
\overline{\text { bit }}_{\text {min }}=\left\lceil\log _{2} \frac{F_{\text {var }}}{2 \xi f_{\text {update }}}\right\rceil,
$$

and the bit rate is given by

$$
\overline{\text { bitrate }}=f_{\text {update }}\left\lceil\log _{2} \frac{F_{\text {var }}}{2 \xi f_{\text {update }}}\right\rceil \text { bps. }
$$

We note that this incremental delivery of feedback information results in significant savings of overhead bandwidth.

\section{Simulations}

We simulated the power control scheme developed in MATLAB. The cost function for the $i^{\text {th }}$ user was chosen as

$$
J_{i}\left(x_{i}, \mathbf{x}_{-i}, h_{i}\right)=\frac{1}{2} \alpha_{i} x_{i}^{2}-u_{i} \log \left(\gamma_{i}+1\right),
$$

where $\alpha_{i}>0$ and $u_{i}>0$ are user specific pricing and utility parameters respectively. Notice that the quadratic pricing and logarithmic utility functions in (11) satisfy assumptions $A 1$, $A 2, A 3$ ', A4 with an appropriate choice of parameter values. Therefore, results of Theorem III.5 apply to the following power update algorithm of the $i^{t h}$ mobile,

$$
\dot{p}_{i}=\lambda_{i} \frac{u_{i}}{p_{i}+\frac{1}{L h_{i}}\left(\sum_{j \neq i, j \in \mathcal{M}_{l}} h_{j} p_{j}+\sigma^{2}\right)}-\lambda_{i} \alpha_{i} h_{i} p_{i},
$$

if $A 5$ and condition on average dwell-time are satisfied. In the simulations, a discretized version of this update scheme was implemented:

$$
\begin{aligned}
p_{i}^{(n+1)}= & p_{i}^{(n)}+\frac{u_{i}}{p_{i}^{(n)}+\frac{1}{L h_{i}}\left(\sum_{j \neq i, j \in \mathcal{M}_{l}} h_{j} p_{j}^{(n)}+\sigma^{2}\right)} \\
& -\alpha_{i} h_{i} p_{i}^{(n)} .
\end{aligned}
$$

The scenario we adopted is the following. We have a simple multicell wireless network consisting of six rectangular shaped cells with 40 users. Base stations are located in the center of each cell. The system parameters are chosen as $L=64$ and $\sigma^{2}=1$. Cost parameters are the same for all users, $u_{i}=$ $10, \alpha_{i}=1, \lambda_{i}=1 \forall i$, and they are fixed for the duration of the simulation. Mobiles are initially located randomly in the system, and their movement is modeled as a random walk with a speed of 0.002 units per update, where set the update frequency to $100 \mathrm{~Hz}$. Hence, if we assume a system with unit size of $100 \mathrm{~m}$, then mobiles move with a speed of $20 \mathrm{~m} / \mathrm{s}$.

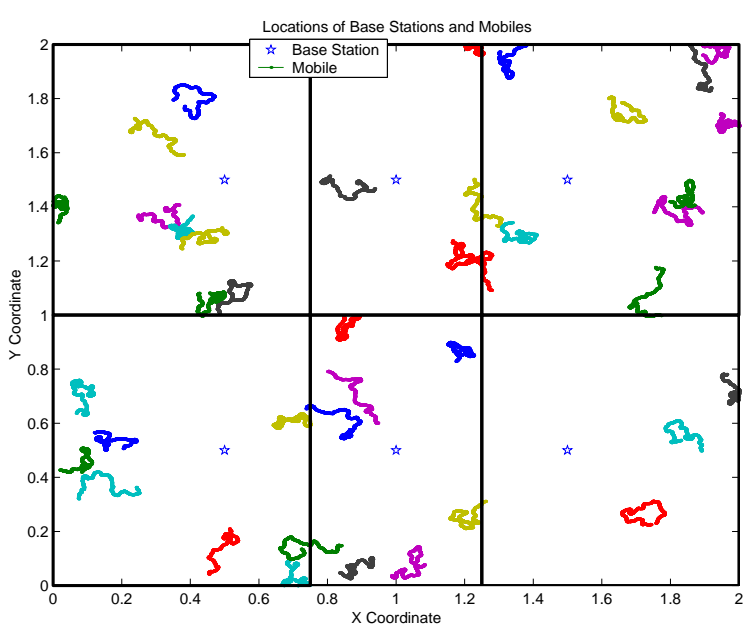

Fig. 3. Locations of base stations and the paths of mobiles.

The channel gain of the $i^{t h}$ mobile is determined by a simple large-scale path loss formula $h_{i}=\left(0.01 / d_{i}\right)^{2}$ where $d_{i}$ denotes the distance to the BS, and the path loss exponent is chosen as 2 corresponding to open air path loss. However, 
fast and random movement of mobiles result in higher variations in the channel gains, and hence, compensate for this simplification.

We use the channel gain as the handoff criterion. Each mobile connects to the base station with highest channel gain, which in turn corresponds to the nearest one. In Figure 3, locations of base stations and the paths of all mobiles in the network are shown. A sample path of a single mobile is shown in Figure 4.

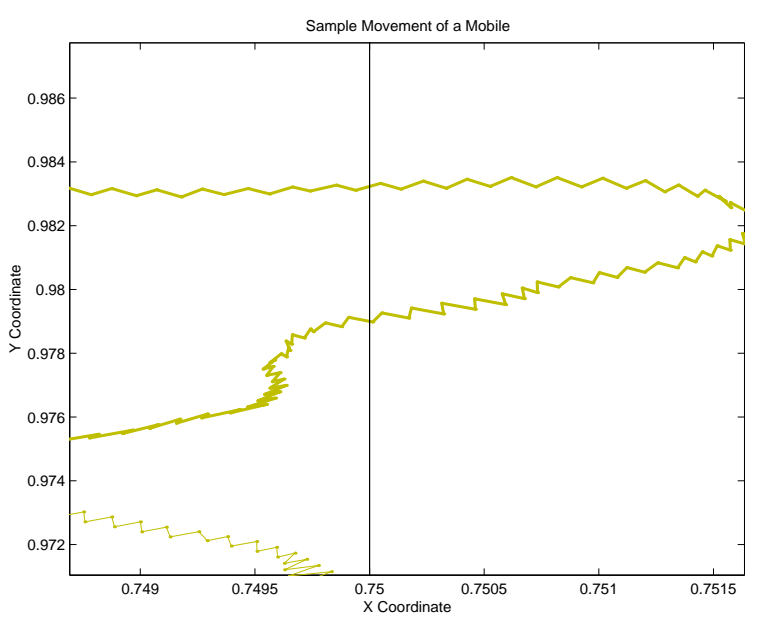

Fig. 4. A sample path of a mobile.

Figures 5 and 6 depict the power levels and SIR values of mobiles for the duration of the simulation. Notice that the power levels converge to the equilibrium points, which shift due to handoffs in the system. Jumps in SIR values can be observed in Figure 6 when a mobile moves from a less congested cell with a smaller number of mobiles to a more congested one or vice versa. Variations in congestion levels in the cells and in channel gains are also the reasons why not all mobiles have the same SIR levels despite having the same cost parameters.

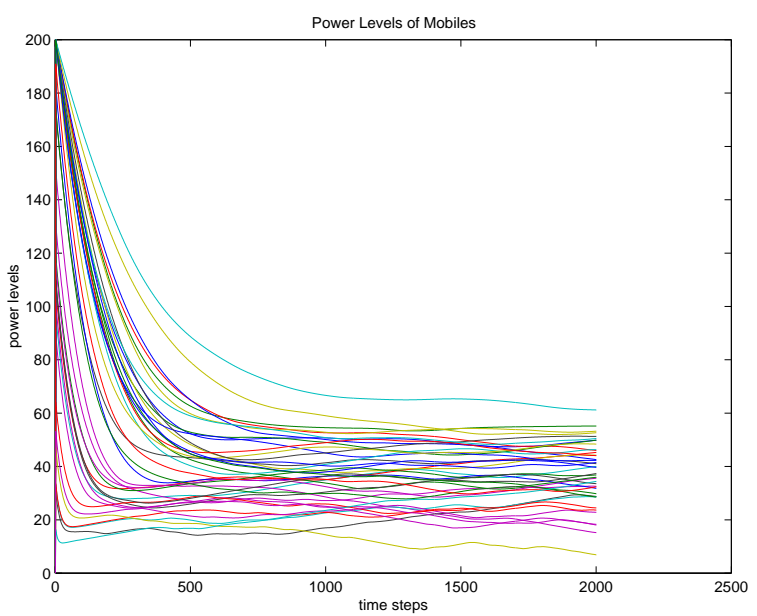

Fig. 5. Power levels of mobiles with respect to time.

The simulation was repeated with the same setup but with a 50 steps ( 0.5 seconds) communication delay between the base station and mobiles. In accordance with the results in Section III-C, convergence characteristics of the system is not significantly affected by the presence of feedback delay except for the convergence rate, as it can be seen in Figure 7. Figure 8 shows the aggregate received power levels at the base stations. The 'relative' smoothness of these values indicate significant savings in system overhead when the feedback quantization scheme analyzed in Section III-D is implemented.

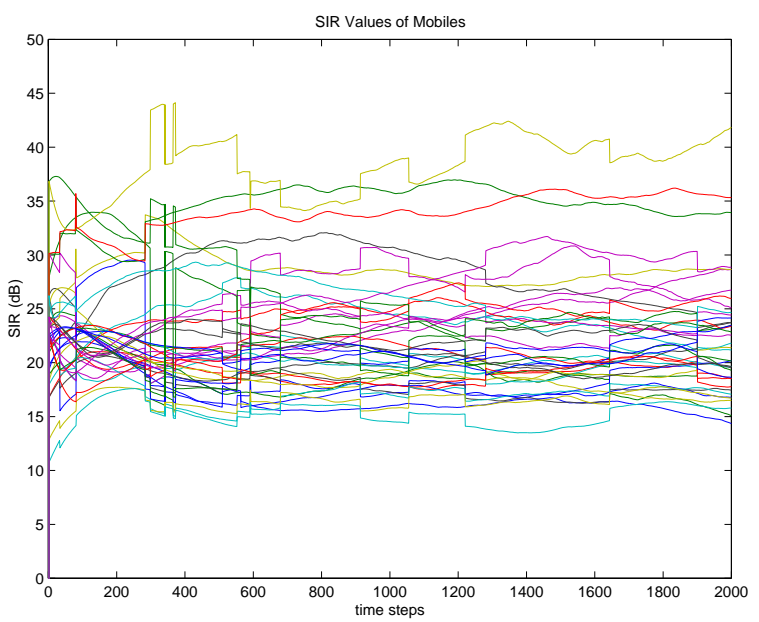

Fig. 6. SIR values of mobiles (in $\mathrm{dB}$ ) with respect to time.

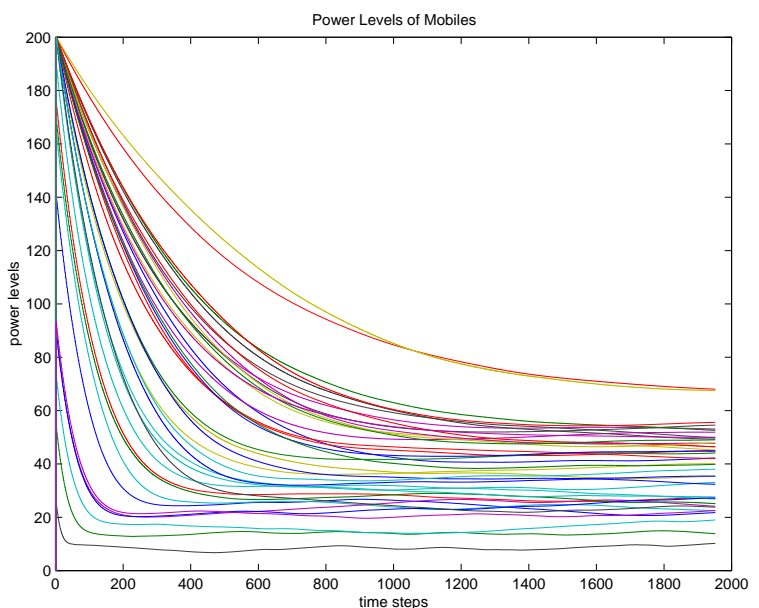

Fig. 7. Power levels of mobiles with respect to time under a communication delay of 50 steps.

\section{CONCLUSION}

In this paper, we have formulated a noncooperative power control game in a multicell CDMA wireless network, which is modeled as a switched hybrid system where handoffs of mobiles between different cells correspond to discrete switching events between different subsystems. Under a set of sufficient conditions, we have shown the existence and global exponential stability of a unique Nash equilibrium for each subsystem under a gradient algorithm. We have also established the global convergence of the dynamics of the multicell 


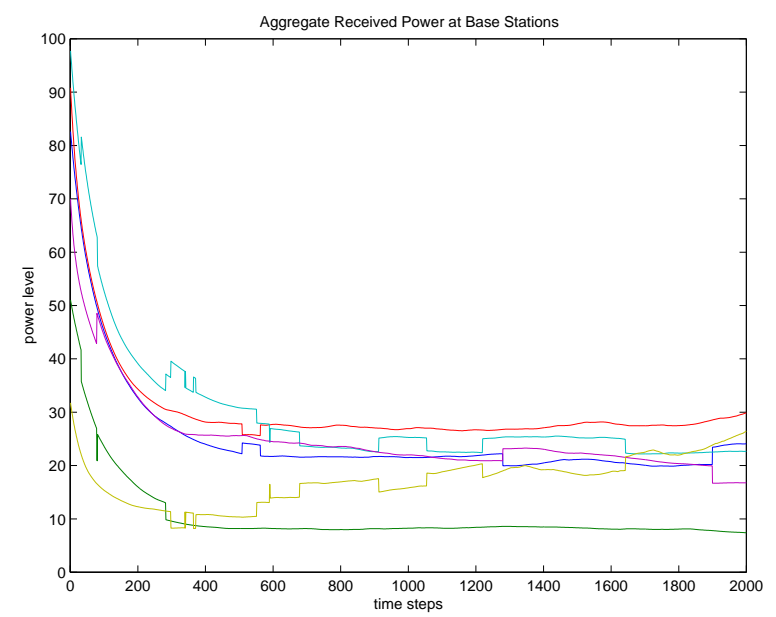

Fig. 8. Aggregate received power levels at the base stations.

power control game to a convex superset of Nash equilibria for any switching (handoff) scheme satisfying a mild condition on average dwell-time. We have investigated the robustness of these results to communication constraints, such as feedback delays and quantization, and have presented a scheme to reduce the communication overhead between mobiles and the base stations. We have also illustrated the proposed power control scheme through MATLAB simulations.

The mathematical model developed captures a fairly broad class of convex cost functions, and addresses the multicell resource allocation problem in CDMA wireless networks. The proposed gradient update algorithm is market-based, distributed in nature, robust with respect to feedback delays, and requires little overhead in terms of system resources. These theoretical results are also supported through realistic simulations. Some directions for future research, which can be viewed as extensions of the present work, are theoretical analysis of the variations in channel gains, and the effect of discretization on the proposed update scheme.

\section{REFERENCES}

[1] D. Falomari, N. Mandayam, and D. Goodman, "A new framework for power control in wireless data networks: Games utility and pricing," in Proc. Allerton Conference on Communication, Control, and Computing, Illinois, USA, September 1998, pp. 546-555.

[2] C. U. Saraydar, N. Mandayam, and D. Goodman, 'Pareto effi ciency of pricing-based power control in wireless data networks," WCNC, 1999.

[3] H. Ji and C. Huang, "Non-cooperative uplink power control in cellular radio systems," Wireless Networks, vol. 4(3), pp. 233-240, April 1998

[4] P. Marbach and R. Berry, 'Downlink resource allocation and pricing for wireless networks," in IEEE Infocom, New York, NY, June 2002.

[5] V. A. Siris, "Resource control for elastic traffi c in cdma networks," in ACM MOBICOM, Atlanta, GA, September 2002.

[6] C. U. Saraydar, N. Mandayam, and D. Goodman, 'Pricing and power control in a multicell wireless data network," IEEE Journal on Seleceted Areas in Communications, pp. 1883-1892, October 2001.

[7] J. Blom and F. Gunnarsson, Power Control in Cellular Radio Systems, Ph.D. thesis, Linkoepings Universitet, EE, Linkoeping, Sweden, 1998.

[8] T. Başar and G. J. Olsder, Dynamic Noncooperative Game Theory, 2nd ed. Philadelphia, PA: SIAM, 1999.

[9] T. Alpcan, T. Başar, R. Srikant, and E. Altman, 'CDMA uplink power control as a noncooperative game," Wireless Networks, vol. 8, pp. 659669, November 2002.
[10] T. Alpcan and T. Başar, "A game-theoretic framework for congestion control in general topology networks," in Proc. of the 41st IEEE Conference on Decision and Control, Las Vegas, NV, December 2002, pp. 1218-1224.

[11] D. Liberzon, 'Hybrid systems and control," Class Notes, University of Illinois at U-C, Illinois, USA, 2002.

[12] J. P. Hespanha and A. S. Morse, 'Stability of switched systems with average dwell-time," in Proc. of the 38th IEEE Conference on Decision and Control, Phoenix, AZ, December 1999, pp. 2655-2660. 